\title{
Two-Echelon Routing Problem for Parcel Delivery by Cooperated Truck
}

\author{
and Drone \\ Yao Liu ${ }^{\mathrm{a}}$, Jianmai Shi ${ }^{\mathrm{a} *}$, Guohua $\mathrm{Wu}^{\mathrm{b}}$, Zhong Liu ${ }^{\mathrm{a}}$, Witold Pedrycz ${ }^{\mathrm{c}}$ \\ a Science and Technology on Information Systems Engineering Laboratory, College of System Engineering, \\ National University of Defense Technology, Changsha, 410073, P. R. China. \\ ${ }^{b}$ School of Traffic and Transportation Engineering, Central South University, Changsha, 410075, China. \\ ${ }^{c}$ Department of Electrical \& Computer Engineering, University of Alberta, Edmonton, AB T6R 2V4 Canada \\ *Corresponding author, jianmaishi@gamil.com.
}

\begin{abstract}
A new variant of two-echelon routing problem is investigated, where the truck and the drone are used to cooperatively complete the deliveries of all parcels. The truck not only acts as a tool for parcel delivery, but also serves as a moving depot for the drone. The drone can carry several parcels and take off from the truck, while returning to the truck after completing the delivery. The energy consumption model for the routing process of the drone is analyzed, when it is utilized to deliver multiple parcels. A two-stage route-based modelling approach is proposed to optimize both the truck's main route and the drone's adjoint flying routes. A hybrid heuristic integrating nearest neighbor and cost saving strategies is developed to quickly construct a feasible solution. The simulated annealing algorithm is applied to improve the quality of the solution, where a Tabu list is employed to improve the search efficiency. Random instances at different scales are used to test the performance of the proposed algorithm. A case study based on the practical road network in Changsha, China, is presented, through which the sensitivity analysis is conducted with respect to some critical factors.
\end{abstract}

Keywords: two-echelon routing; vehicle routing; truck and drone; heuristic; simulated annealing algorithm 


\section{Introduction}

Over the years, the full-blooming E-commerce has incentivized the express delivery to skyrocket. It is expected to distribute goods with shorter delivery time and lower courier cost, which throws out many challenges to the last mile delivery. Traditional distribution based on ground vehicles can hardly satisfy the customers for their increasing requirement on shorter delivery time, as they expect to receive their parcels in one or two days after they complete the online orders. Ground delivery vehicles are limited by the established infrastructure and geographical obstacles such as roads and rivers, which would vastly add to the distribution time and operating costs. A large number of ground vehicles bring about atmospheric pollution and traffic jam in urban areas, which is certainly against the public wish for the environmental protection. These problems drive many researchers to look for more efficient delivery ways and thus show great interests in drone delivery.

Delivery by unmanned aerial vehicles (UAVs), or drones, has been feasible due to the rapid development of automation and artificial intelligence technologies. In general, drones can significantly decrease the delivery cost and time, as they can fly directly to recipients with being less blocked by obstacles and not considering ground traffic condition. Besides, drones are more environment-friendly with lower power consumption and less air pollution. Thus, realizing these advantages, many companies have investigated drone delivery problems. In addition to the first few companies applying UAVs, such as Amazon, DHL and Google (Murray and Chu, 2015; Ha et al., 2018), JD has also tried the drone delivery in several provinces in China. Alibaba Group and Baidu also utilize the parcel copter for takeout. However, depending upon the characteristics of drones, it may not be optimal to deliver-by-drone to all customers. Restricted by short endurance and low load capacity, drones have limited flight time and can only carry small packages.

Considering these practical difficulties, both trucks and drones have their own limitations and advantages, which are summarized in Table 1. Nevertheless, if they work together, there are several advantages due to their strong complementarity. Primarily, the effective delivery range of drone is enlarged, when the ground vehicle acts as a moving depot for carrying the drone. With longer travelling distance and larger load capacity, ground vehicles can serve dual roles as both a mobile depot and a delivery resource. Furthermore, drones are less limited by the ground traffic and can perform better in some areas hard for trucks to reach, such as some places with traffic congestion or inconvenient 
transportation. As for the drone's short endurance, it can be reused with replacing the battery or charging on the ground vehicles. Thus, the combination of drone and truck has promised a bright prospect. Some companies have applied this interesting approach. Mercedes-Benz released a conceptual car called "Vision Van" in September 2016. Equipped with a fully automated cargo loading system, this van deploys the delivery drone to get parcels to customers quickly. As described, it can significantly reduce delivery time, and increase efficiency. In addition, UPS also develops a project named "Last Mile" to deploy UAVs on a mobile truck, and a trial run in Florida was conducted in February 2017.

Table 1. Comparison of drone and ground vehicle for delivery

\begin{tabular}{lll}
\hline & Drone & Ground Vehicle \\
\hline Load capacity & Small & Large \\
Endurance range & Short & Unlimited \\
Cost per kilometer & Low & High \\
Carbon emission & Low & High \\
Traffic & No impact & Traffic jam \\
Route & Direct in air & Along road network \\
\hline
\end{tabular}

There has been some pioneering work for investigating the parcel delivery problem for cooperated truck and drone, e.g. Murray and Chu (2015), Ha et al. (2017) and Agatz et al. (2018). These works studied the cooperated routing problem through extending the classical travelling salesman problem, and also assume that the drone can only take one parcel in each flight. However, the drone can carry several small parcels and complete their delivery in one flight, as the investigation in Dorling et al. (2017). When the drone can take multiple parcels, the routing problem for the cooperated truck and drone becomes a new variant of two-echelon routing problem as shown in Figure 1, in which more new challenges are confronted.

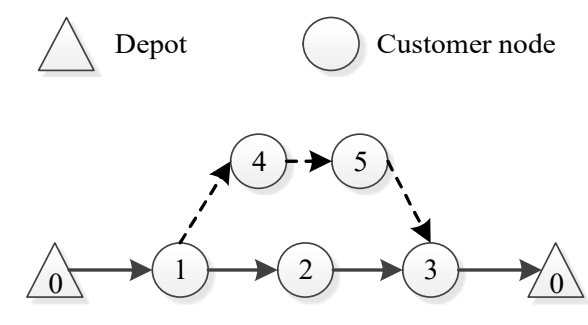

(a)

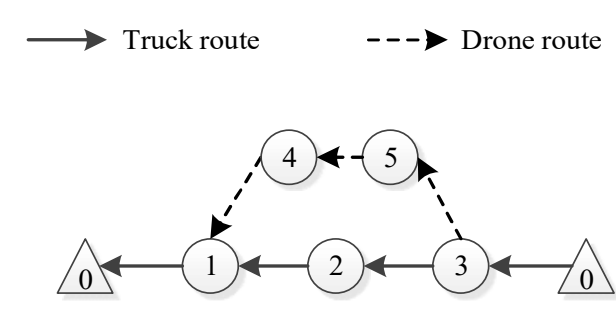

(b)

Figure 1. Two solutions with different directs for an example with five customer nodes

In the two-echelon routing problem for the truck and drone (2E-RP-T\&D), it is usually 
assumed that the drone can take off and land on the truck at any customer node where the truck stops. Also, the second echelon route of the drone is open, which is different from common two-echelon routing problems. Both distinguishing features make the problem more complex.

In the parcel delivery process of the drone carrying multiple parcels, not only the payload capacity and the endurance capacity must be considered, but also the energy consumption process of the drone's battery should also be considered. The energy consuming rate is a piecewise function depending on the sequence of parcels' delivery, and the energy consumed is quite different even for two routes with the same length while different directions. For example, in Figure 1, if the package of Customer 4 is heavier than that of Customer 5, it is better for the drone to first serve Customer 4 to unload the heavy cargo. However, when the drone travels in the contrary direction, the heavy payload would consume more energy on the route of $3 \rightarrow 5 \rightarrow 4$, which causes more cost. Therefore, with the directivity of the paths in consideration, the search space of 2E-RP-T\&D is further enlarged, compared to traditional two-echelon routing problems.

Motivated both by the adaption of new technologies and delivering tool (drone) in practical transportation industry, and the theoretical gap existing in the current literature, a new twoechelon routing problem for the cooperated truck and drone is studied. As far as we know, it is the first time to study the truck \& drone cooperatively routing problem simultaneously considering the following two aspects: (1) the drone can deliver multiple parcels in a single flying route, and (2) the effect of varying payload on energy consumption is considered. To formulate the problem a two-stage route-based modelling approach is proposed, where the routes for the truck and drone are optimized. Due to the complexity of the problem, a hybrid heuristic through integrating the nearest neighbor and cost saving strategies are developed to construct a feasible solution in short time, and the simulated annealing algorithm combined with the Tabu list is proposed to further improve the quality of the solution. Both random instances and practical case are used to test the proposed algorithms, and computational results show that our approach can efficiently solve the problem.

The paper is structured as follows. Section 2 presents the literature review, and Section 3 illustrates the problem and the model. Section 4 introduces the heuristic algorithms. The experiment for random instances is carried out and sensitivity analysis is conducted with an actual case in Section 5. At last, Section 6 presents the conclusion and outlines future works. 


\section{Literature Review}

Due to the high efficiency and low cost, the drone presents huge application opportunities in various industries in recent years. One of the applications is parcel delivery which has been investigated in many researches. Sundar and Rathinam (2014) consider a single drone routing problem with multiple depots available for refueling the drone and minimize the total fuel the drone required for visiting all the targets. San et al. (2016) propose the implementation steps of assigning multiple drones to effectively deliver items to target locations. The actual operation of autonomous deliveries is simplified and a Genetic Algorithm is proposed to solve the problem. There is also a research on the automated drone delivery system (Choi and Schonfeld, 2017), in which drones can lift multiple packages within a service area of given radius. Numerical analysis is applied to minimize the total costs by exploring the relationship among four parameters: working period, drone speed, demand density of service area, and battery capacity. Furthermore, multiple vehicles and time windows are considered in Ham (2018), whose study extends the problem by considering the drone can either fly back to depot for next delivery or fly directly to another customer for pickup.

With regards to the military applications, unmanned combat aerial vehicles are considered to destruct predetermined targets with munitions, which has the limitations of the lower bound and upper bound (Shetty, et al., 2008). Besides, drones can gather intelligence information from a set of known targets after equipped with sensors. Avellar et al. (2015) solve the problem of minimum time coverage of ground areas using a group of drones equipped with image sensors. Persistent Intelligence, Surveillance and Reconnaissance (PISR) routing problem are also considered by minimizing the time of delivering the collected data to the control station (Manyam et al., 2017).

In the works mentioned above, the endurance capacity is set as a fixed value. However, based on the actual situation, the effect of the payload weight on energy consumption of the drone during the flight should not be ignored. There are several studies mentioned the relationship between the endurance and payload weight. Mufalli et al. (2012) consider the surveillance mission in which the available flight time would be reduced after attaching a sensor. Dorling et al. (2017) propose two vehicle routing problem (VRP) variants for drone delivery. 
Calculated with a linear approximation function, the energy consumption varies linearly with payload and battery weight. Besides, Song et al. (2018) mainly focus on the characteristics of the UAV logistics system and discuss the effect of cargo weight on flight ability. However, in these problems, only drones are applied for the delivery, and the truck is not included.

Since the combination of drone and truck can greatly improve the delivery efficiency, we are aware of some works investigating the problem. Murray and Chu (2015) introduced the problem, "Flying Sidekick Traveling Salesman Problem" (FSTSP), which proposes a mixed integer liner programming formulation and a heuristic. In detail, the heuristic is based on the idea of "Truck First, Drone Second", in which they first construct a truck-only route and iteratively replace the truck nodes by drone nodes to reduce the objective value. Recently, this problem is also solved by some variable neighborhood heuristics (Freitas and Penna, 2018), which perform much better on the large-scale instances. There are also a slightly different problem - called "Traveling Salesman Problem with Drone” (TSP-D) (Agatz et al., 2018), in which the drone has to follow the same road network as the truck. This problem is also modelled as a MILP formulation and solved by a heuristic based on local search. Furthermore, some variants of this problem are also explored. Ha (2018) introduces a time span into the TSP-D, which the drone and the truck can wait for each other with the limited waiting time. Besides, two heuristics of two concepts: "Drone First, Truck Second" (DFTS) and "Truck First, Drone Second" (TFDS) are designed. Different from the former paper with the objective of minimizing the cost, the author also tried to reduce the total time in another publication (Ha et al., 2015). However, in these works, only one customer's parcel can be arranged on the drone in each flying route.

Cooperation between drone and truck is also widely used for surveillance and mapping. There is a research exploiting the cooperative air and ground surveillance, in which the framework and algorithm are proposed for search and localization (Grocholsky et al., 2006). Additionally, with the restriction of the UAV's fuel capacity, Maini et al. (2015) consider the refueling Unmanned Ground Vehicle (UGV) for successful mission completion. Savuran and Karakaya (2015) propose a route optimization method for a carrier-launched UAV. In this problem, the UAV visits the targets to execute the mission while the carrier keeps on moving on its own route. Furthermore, a similar routing problem is investigated (Luo et al., 2017) and 
the difference is that the GV travels on the road network while its UAV visits the targets beyond the road. Although these problems all investigate the routing problem in the combination of trucks and drones, the payload of the drone stays the same while visiting multiple targets in one route, in which the relationship between payload and energy consumption would not be involved.

From the above review, we can see that a new variant of the classical vehicle routing problem has not been studied, which is the two-echelon routing problem for cooperated truck and drone, especially when multiple parcels can be delivered in one drone's flying route and the effect of varying payload on energy consumption is considered. Therefore, this new problem is studied in this paper. The energy consumption model of the drone during the parcel delivering process is analyzed, and a mathematical model is developed to optimize both the truck and drone's routes in the next section.

\section{Problem Description and Model Development}

\subsection{Definitions and notations}

In order to facilitate the model formulation, several definitions are first introduced as follows:

Directed main-route is a closed path traveled by the truck starting from the depot for visiting some customer nodes and returning to the depot, e.g. $(0,1,2,3,0)$ shown in Figure 1(a).

Directed sub-route is path segment of a directed main-route. For example, there are ten directed sub-routes in the directed main-route, $(0,1,2,3,0)$, in Figure 1a, which are $(0,1),(1,2)$, $(2,3),(3,0),(0,1,2),(1,2,3),(2,3,0),(0,1,2,3),(1,2,3,0)$ and $(0,1,2,3,0)$.

Adjoint sub-route is directed path including one or several customer nodes that can be visited by the drone when the truck travels a corresponding sub-route. For example, in Figure 1a, $(1,4,5,3)$ is an adjoint sub-route of directed sub-route $(1,2,3)$, which means the drone takes off from the truck at node 1 , conducts the delivery tasks of nodes 4 and 5, and then returns to the truck at node 3 . Also, sub-routes $(1,4,3)$ and $(1,5,3)$ are also potential adjoint sub-routes of directed sub-route $(1,2,3)$.

The notations used in the problem description and model development are presented as follows. 


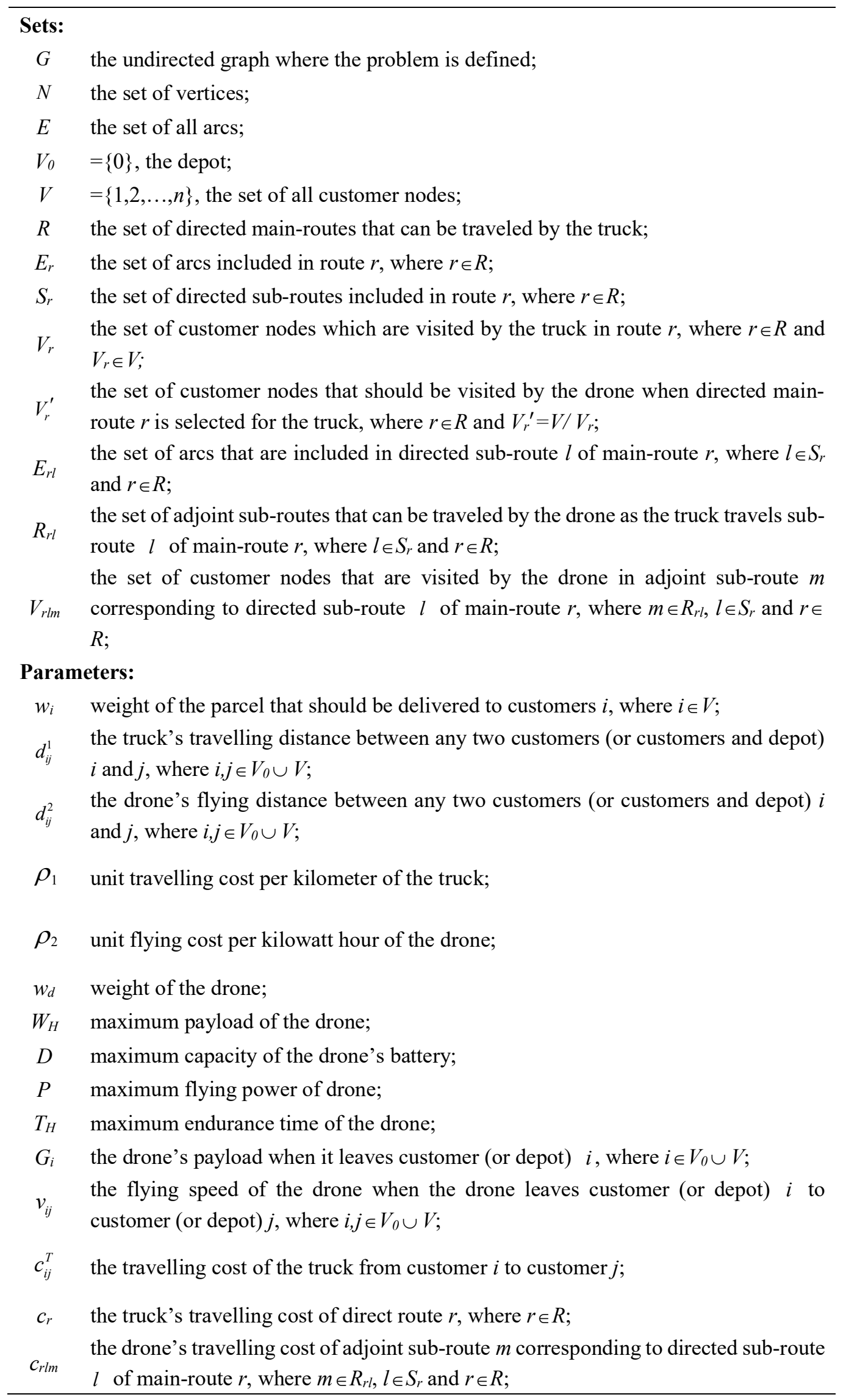


$\begin{array}{ll}a_{i j r l} & 1 \text { if } \operatorname{arc}(i, j) \in E_{r l}, 0 \text { otherwise, where } i, j \in V_{r}^{\prime}, l \in S_{r} \text { and } r \in R \\ b_{i r l m} & 1 \text { if } i \in V_{r l m}, 0 \text { otherwise, where } i \in V_{r}^{\prime}, m \in R_{r l}, l \in S_{r} \text { and } r \in R\end{array}$

Variables:

$x_{r} \quad 1$ if directed main-route $r$ is selected for the truck, 0 otherwise, where $r \in R$;

1 if adjoint sub-route $m$ corresponding to directed sub-route $l$ in directed main-route

$y_{r l m} \quad r$ is selected for the drone, 0 otherwise, where $m \in R_{r l}, l \in S_{r}$ and $r \in R$.

\subsection{Problem description}

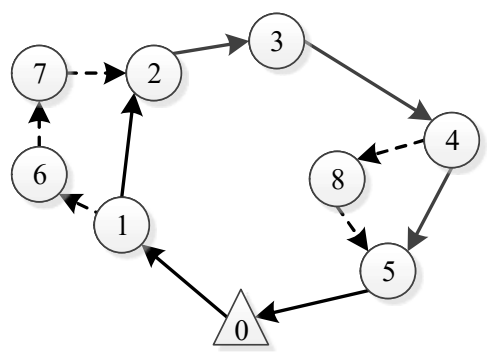

Figure 2. An illustration of the solution for an example 2E-RP-T\&D

In the two-echelon routing problem for truck and drone (2E-RP-T\&D), it is assumed that the capacity of the truck is always sufficient for all parcels, and thus can be viewed as uncapacitated. The payload capacity of the drone is limited and known. The problem aims at finding the optimal directed main-route of the truck and a set of directed adjoint sub-routes of the drone to complete the delivery of all parcels, while the drone's capacity constraints on payload and battery are not violated. Figure 2 shows a feasible solution for an example of 2ERP-T\&D. The route belonging to the first echelon is traveled by the truck, represented as solid lines, whereas the drone routes, depicted as dashed lines, belong to the second echelon.

More formally, the 2E-RP-T\&D can be described as follows. An undirected graph $G=(N$, $E$ ) is defined. Set $N=V_{0} \cup V$ is the set of vertices, where $V_{0}=\{0\}$ represents the depot and $V$ $=\{1,2, \ldots, n\}$ is the set of all customer nodes. Set $E$ is the set of edges that can be traveled by the truck or the drone.

The truck starts from the depot, taking all the parcels and the drone. The truck can only travel on the road network and deliver the parcel to its customer. When the truck delivers the parcel, the drone can also carry some small parcels and take off from the truck to complete some deliver tasks simultaneously. We assume that the drone can only take off / land on the truck when it stops at customer nodes (or depot), where the drone can be charged or change the 
battery. After completing the delivery of all parcels, the truck must return to the depot with the drone. The objective is to find the main-route for the truck and all the adjoint sub-routes for the drone to complete the delivery of all parcels and minimize the overall travelling cost of the truck and drone.

\subsection{The energy consuming process for the drone's battery in an adjoint sub-route}

The weight of each parcel cannot be neglected compared to the self-weight of the drone. The energy consumption rate of the drone depends on its self-weigh and the weight of the parcels it carried, which would become smaller step by step once the parcels have been dropped off at the customer nodes. The energy consumption model for an adjoint sub-route with multiple customer nodes is important for estimating the cost of this sub-route.

After selecting the adjoint sub-route and assigning the delivery tasks, the drone would load all the corresponding parcels in order. Then it would travel to visit the assigned customers and unload the packages one by one, which means that the payload would show a phased reduction. Since the energy consumption rate can be viewed to vary linearly with payload (D'Andrea, 2014; Dorling et al., 2017), the shape of the function for estimating this rate would show a piece-wise form, which becomes lower step by step as the parcels are delivered to the customers. Figure 3(a) presents an illustration of changes on the drone's battery power in a sub-route with 3 parcels, while Figure 3(b) displays the changes on the battery remaining capacity in this route.

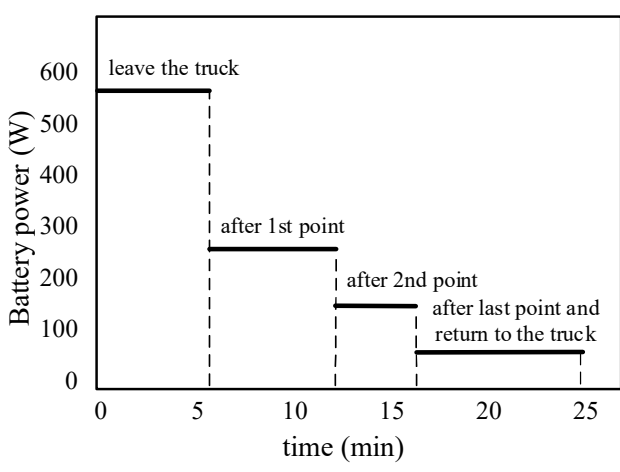

(a) The changes of the battery power in the flying process

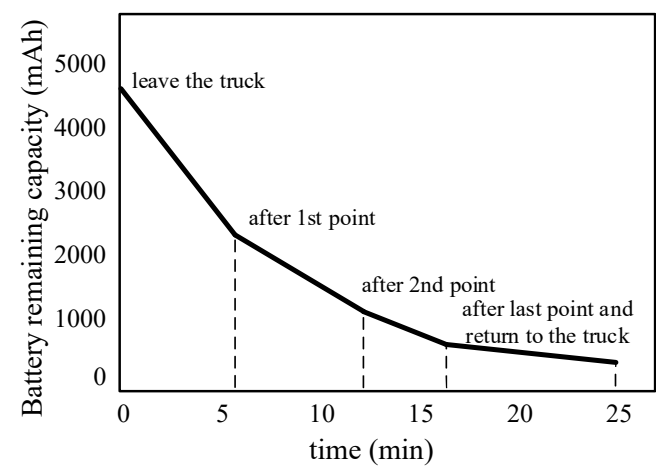

(b) The changes of the battery remaining capacity in the flying process

Figure 3. The illustration of the energy consumption process for the drone in a sub-route with 3 parcels

When leaving customer (or the depot) $i$ to customer (or the depot) $j\left(i, j \in V_{0} \cup V\right.$ ), we assume that the drone has the self-weight $w_{d}$ and a payload $G_{i}$. Besides, the flying speed of the drone is 
denoted as $v_{i j}$. Then in the path segment from customer $i$ to customer $j$, the battery power $p_{i j}$ can be represented as follows.

$$
p_{i j}=\frac{\left(w_{d}+G_{i}\right) v_{i j}}{370 \eta \gamma}+e,
$$

Where $\eta$ is the conversion efficiency of the motor, $\gamma$ is the lift ratio, and e is the energy loss of the drone battery (D'Andrea, 2014).

Then the flying time from customer $i$ to customer $j$ and the corresponding energy consumption is as shown below.

$$
\begin{aligned}
& t_{i j}=\int_{0}^{d_{i j}} \frac{1}{v_{i j}} d s \\
& W_{i j}=\int p d t=\left[\frac{\left(w_{d}+G_{i}\right) v_{i j}}{370 \eta \gamma}+e\right] \int_{0}^{d_{i j}} \frac{1}{v_{i j}} d s
\end{aligned}
$$

When the brand and type of the drone is pre-determined, the value of all the related parameters in (1)-(3) are known. Usually, the drone has a maximum power, $P$, due to the limitations of battery and motor. In order to obtain the highest delivery efficiency, we assume that the drone would maintain its maximum power during the flight, which means

$$
p_{i j}=P
$$

Thus, based on the above assumption, the flying speed and time from customer $i$ for customer $j$ can be estimated as presented below.

$$
\begin{aligned}
& v_{i j}=\frac{370 \eta \gamma(P-e)}{w_{d}+G_{i}} \\
& t_{i j}=\frac{d_{i j}\left(w_{d}+G_{i}\right)}{370 \eta \gamma(P-e)}
\end{aligned}
$$

And the relationship between payload and energy consumption can be expressed as Equation (7), which is the energy consumption model.

$$
W_{i j}=P t_{i j}=\left(w_{d}+G_{i}\right) \frac{d_{i j} P}{370 \eta \gamma(P-e)}
$$

Based on the above expressions, we can calculate the overall amount of energies consumed by the drone in an adjoint sub-route, and thus judge if it is over the capacity of the battery. Also, the 
cost of the adjoint sub-route can be obtained.

\subsection{Model development}

When multiple parcels are delivered by the drone in a single flight, the model development for the two-echelon routing problem becomes quite difficult. Thus, to offer some more precisely understanding on the problem definition, we presented a two-stage route-based mathematical formulation. Given the network $G=(N, E)$, we assume that all possible directed main-routes of the truck can be enumerated and let $R$ be the set of these main-routes. Each route $r \in R$ starts from the depot $V_{0}=\{0\}$, visits one or several customers in $V$, and return to the departure, e.g. $(0,1,2,3,4,5,0)$ in Figure 2. For any directed main-route $r(r \in R)$, its cost can be calculated and noted as $c_{r}$. When route $r(r \in R)$ is selected for the truck, let $V_{r}$ is the set of customers that visited by the truck and $V_{r}^{\prime}$ is the set of drone customers. Also, we assume that all the directed subroute in a given main-route $r(r \in R)$ can be enumerated and let $S_{r}$ be the set of all sub-routes in main-route $r$. Similarly, let $E_{r l}$ be denoted as the set of arcs that are included in directed subroute $l$ and $R_{r l}$ be denoted as the set of all possible adjoint sub-routes that can be traveled by the drone as the truck travels sub-route $l$ in route $r$. Each adjoint sub-route $m\left(m \in R_{r l}\right)$ starts from the first customer of the corresponding sub-route $l$ and ends at the last customer of $l$ after visiting one or several customers in $V_{r}^{\prime}$. Besides, $V_{r l m}$ is the set of customer nodes that are visited by the drone in adjoint sub-route $m\left(m \in R_{r l}\right)$ corresponding to directed sub-route $l$ in route $r$. And according to the energy consumption model, the drone's travelling cost of adjoint sub-route $m$ can be calculated and denoted as $c_{r l m}$.

Let $x_{r} \in\{0,1\}$ be a binary variable equal to 1 if directed route $r(r \in R)$ is selected for the truck, and 0 otherwise. Given a truck route $r \in R$ and a directed sub-route $l \in S_{r}$, let $y_{r l m} \in\{0,1\}$ be a binary variable equal to 1 if adjoint sub-route $m\left(m \in R_{r l}\right)$ is selected for the drone, and 0 otherwise. Furthermore, given two customers $i, j\left(i, j \in V_{0} \cup V\right)$ and an adjoint sub-route $m(m \in$ $\left.R_{r l}\right)$, let $a_{i j r l} \in\{0,1\}$ be a binary parameter equal to 1 if arc $(i, j) \in E_{r l}$ and 0 otherwise, let $b_{i r l m}$ $\in\{0,1\}$ be a binary parameter equal to 1 if $i \in V_{r l m}$ and 0 otherwise.

Based on the above assumptions and definitions, we develop a two-stage route-based mathematical formulation for $2 \mathrm{E}-\mathrm{RP}-\mathrm{T} \& \mathrm{D}$. In the first stage, the model for optimizing the drone's adjoint sub-routes for a given main-route of the truck is proposed, which can be used 
to calculate the minimum cost of the drone for all the possible main-routes in $R$. In the second stage, the overall cost for both truck and drone is optimized.

\subsubsection{Model 1: Optimizing the drone's adjoint sub-routes}

For any given truck route $r(r \in R)$, the sets, $E_{r}, V_{r}$ and $V_{r}^{\prime}$, are determined. On this basis, all possible and feasible drone adjoint sub-routes corresponding to route $r$ can be known. Then the minimum cost for the drone's adjoint sub-routes to visit all nodes in $V_{r}^{\prime}$ can be calculated through Model 1, which is formulated as follows.

$$
\operatorname{Min} z_{r}=\sum_{l, m} c_{r l m} y_{r l m}
$$

s.t.

$$
\begin{aligned}
& \sum_{l, m} a_{i j r l} y_{r l m} \leq 1, \forall i, j \in E_{r} \\
& \sum_{l, m} b_{i r l m} y_{r l m}=1, \quad \forall i \in V_{r}^{\prime}, \\
& y_{r l m} \in\{0,1\}, \quad \forall m \in R_{r l}, l \in S_{r} .
\end{aligned}
$$

The objective function (8) minimizes the cost of the drone for delivering all the parcels that are not delivered by the truck in route $r$. Given a truck route $r(r \in R)$, the constraint (9) ensures that each path segment of truck route $r$ can only correspond to at most one adjoint sub-route, which avoid intersection among drone routes. Constraint (10) impose that all customer nodes in $V_{r}^{\prime}$ must be visited exactly once. Finally, constraints (11) define the decision variables.

\subsubsection{Model 2: Optimizing the truck's directed main-route}

To solve the problem, we have to enumerate all the possible directed main-routes for the truck, and find the optimal result with the minimum total cost for both the truck and the drone, in which the drone's optimal cost for each truck route $r, z_{r}{ }^{*}$, is calculated by solving Model 1. The model for optimizing the truck's directed main route is noted as Model 2 and presented as follows.

$$
\begin{array}{ll}
\text { Min } & Z=\sum_{r} c_{r} x_{r}+\sum_{r} x_{r} z_{r}^{*} \\
\text { s.t. } & \\
& \sum_{r} x_{r}=1
\end{array}
$$




$$
x_{r} \in\{0,1\}, \quad \forall r \in R \text {. }
$$

The objective function (14) minimizes the total cost given by two main components, which are the truck's travelling cost and the drone's flying cost. $z_{r}{ }^{*}$ is obtained through solving Model 1. Constraints (15) ensure that only one truck route can be selected. Constraints (14) define the decision variables. For some directed main-route $r(r \in R)$, there may be no feasible solution can be obtained in Model 1, and we let $z_{r}{ }^{*}$ to be $+\infty$ in this situation.

We can see that the 2E-RP-T\&D can be solved by utilizing Model 1 and Model 2. First, all the possible directed main-routes of the truck should be enumerated to obtain set $R$. For each main-route $r(r \in R)$, all the adjoint sub-routes should be enumerated and the optimal sub-routes of the drone can be obtained by solving Model 1. Then the optimal solution for minimizing the overall cost of the truck and drone can be calculated by Model 2. However, this approach can only solve problems with a very small number of customer nodes. That is because the 2E-RPT\&D is NP-hard as it generalizes other known NP-hard problems, such as the Capacitated VRP (CVRP). It would become much more difficult to explore the solution space as the size of the instance increases, which means the massive problem cannot be solved by the two-stage approach. Thus, efficient heuristic algorithms have to be developed.

\section{Solution}

This section proposes a solution algorithm based on simulated annealing. An initial solution is first constructed by a new heuristic integrating nearest neighbor and cost saving strategies. Then the Simulated Annealing algorithm combined with a Tabu list is applied to improve the initial solution.

\subsection{Hybrid heuristic integrating nearest neighbor and saving strategies}

The hybrid heuristic is designed based on the idea of "Truck First, Drone Second", which integrates both nearest neighbor and saving strategies. Firstly, Nearest Neighbor Search (NNS) is applied to build a directed main-route for the truck to visit all customer nodes. Then the idea of maximum cost savings is adopted to find the set of the drone's adjoint sub-routes by replacing some truck nodes with drone nodes. 


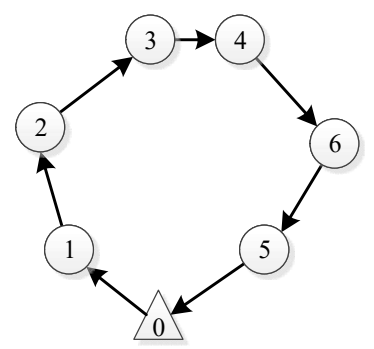

(a) truck-only tour solution solved by NNS

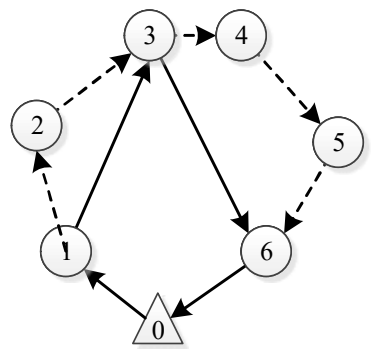

(b) truck \& drone solution by cost saving replace

Figure 4. An illustration of the solution for six customer nodes

\subsubsection{Construct a directed main-route based on nearest neighbor strategy}

The heuristic based on Nearest Neighbor search is a well-known constructive search algorithm, which is one of the earliest methods proposed for solving TSP problems (Cover and Hart, 1967). The nearest neighbor search heuristic is utilized to construct a complete tour for visiting all customers by the truck. It adopts the principle of selecting the next nearest unvisited node until all nodes have been covered. It runs fast, however, the optimality of the tours it produces highly depends on the layout of the customer nodes.

\subsubsection{Construct adjoint sub-routes based on cost saving strategy}

After completing the Nearest Neighbor Search, a directed main-route can be built, which forms a customer list. The weights of the parcels for different customers are different. Some of them are heavy packages that can only be delivered by the truck, while the others are light ones that can be delivered by the drone. For the light parcels in the complete tour obtained in section 4.1.1, if they can be delivered by the drone, we utilize the drone to replace the truck for delivering them. The replacing process is conducted one by one according to a cost saving strategy proposed by Clarke and Wright (1964). The CW algorithm was originally applied to Vehicle Routing Problem (VRP), which aims to find the optimal routes to deliver all the given customers. Its main idea is to combine the two routes into one route under the limitations of the vehicle load and find the maximum distance reduction. Similarly, the algorithm in this paper tries to save the most cost by replacing a truck customer by a drone customer.

Specifically, in order to find the most saving customer in each replacing operation, four conditions are identified according to the delivery ways of the former customer and the latter customer. The four conditions are illustrated in Figure 5. In condition 1, there are three customers visited by the truck in sequence, and the parcel of the middle one (Customer 2) is 
light, whose delivering way can be changed from the truck to drone. The drone would be launched from Customer 1 and return to Customer 3 after serving Customer 2, and a new adjoint sub-route can be generated. Then, the saved cost of this replacement can be calculated as $\left(c_{12}^{T}+c_{23}^{T}\right)-\left(c_{13}^{T}+c_{1-2-3}^{D}\right)$, where $c_{1-2-3}^{D}$ is the flying cost of the adjoint sub-route, $(1,2,3)$.

In condition 2, a truck visiting node, which is also the landing node of a drone's adjoint sub-route, is added to the end of this adjoint sub-route. As illustrated in Figure 5(b), Customer 1 is delivered by the drone while Customer 2 and 3 are delivered by the truck in sequence. At this case, the feasibility should be judged whether the drone can serve Customer 2 after finishing the delivery of Customer 1. Besides the limitation of payload, energy consumption of the whole route needs to be re-calculated according to the method in section 3.3. The same check should also be conducted for the third and fourth condition. If the replacement in condition 2 is feasible, the saved cost can be calculated as $\left(c_{42}^{T}+c_{23}^{T}+c_{\cdots 1-2}^{D}\right)-\left(c_{43}^{T}+c_{\cdots 1-2-3}^{D}\right)$, where $c_{\cdots 1-2-3}^{D}$ is the flying cost of the original sub-route $(---1-2)$ and $c_{\cdots 1-2-3}^{D}$ is the flying cost of the sub-route with Customer 3 added.

In condition 3, a truck visiting node, which is the taking off node of a drone's adjoint subroute, is added to the start of this adjoint sub-route. As illustrated in Figure 5(c), Customer 2 is visited by the truck and is the taking off node of the drone. If the parcel in customer 2 can be delivered by drone and added to the start of the adjoint sub-route, the saved cost can be calculated as $\left(c_{12}^{T}+c_{24}^{T}+c_{2-3 \ldots}^{D}\right)-\left(c_{14}^{T}+c_{1-2-3 \ldots}^{D}\right)$, where $c_{2-3 \ldots}^{D}$ is the flying cost of the original sub-route (2-3---) and $c_{1-2-3 \ldots}^{D}$ is the flying cost of the sub-route with node 1 added.

In condition 4, two drone's adjoint sub-routes are merged, which is illustrated in Figure 5(d). It can be seen that, in Figure 5(d), Customer 2 is visited by the truck, which is the landing node of adjoint sub-route (---1-2) and the taking off node of adjoint sub-route (2-3---). If the two adjoint sub-routes can be merged into one adjoint sub-route, the saved cost can be calculated as $\left(c_{42}^{T}+c_{25}^{T}+c_{\cdots 1-2}^{D}+c_{2-3 \cdots}^{D}\right)-\left(c_{45}^{T}+c_{\cdots 1-2-3 \cdots}^{D}\right)$, where $c_{\cdots 1-2}^{D}$ is the flying cost of the sub-route $\quad(--1-2), \quad c_{2-3 \ldots}^{D} \quad$ is the flying cost of the sub-route $(2-3---)$ and $c_{\cdots 1-2-3 \ldots}^{D}$ is the flying cost of the sub-route (---1-2-3---). 
Based on the above four conditions, check all the nodes visited by the truck, calculate the saved cost for every possible nodes and replace the one with maximum cost saving by drone visiting. Repeat this evaluation and replacing step until no more positive cost saving can be found, which means the total cost cannot be decreased through changing the delivery ways of customers.

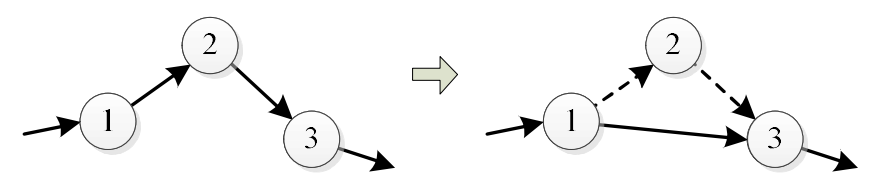

(a) Condition 1: generating a new adjoint sub-route

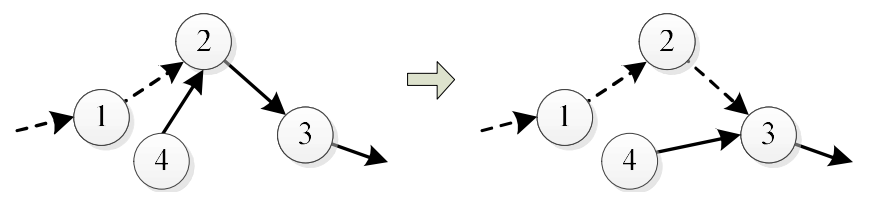

(b) Condition 2: adding a node at the end of a drone's adjoint sub-route

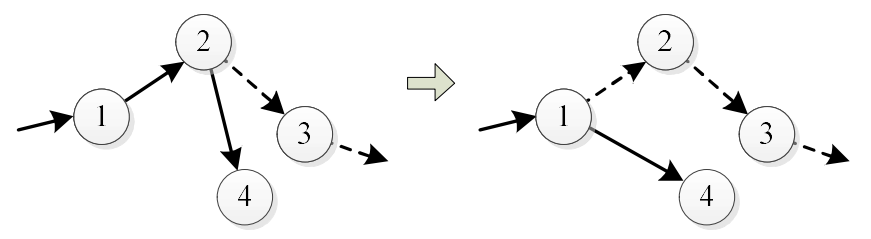

(c) Condition 3: adding a node at the start of a drone's adjoint sub-route

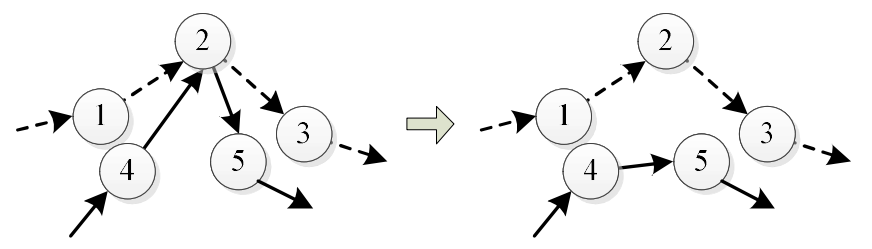

(d) Condition 4: merging two drone's adjoint sub-routes

Figure 5. Four conditions for replacing the truck visiting node by the drone visiting node

\subsubsection{Main optimization procedure}

The main procedure for the hybrid heuristic is shown in Algorithm 1. At first, according to the actual road distance, the truck is assigned to leave from the depot and choose to serve the nearest customer every time until all the parcels haven been delivered. When the truck returns to the depot, a truck-only route is generated (Line 1). Then loops would be executed following the sequence of the customer list in this main route. In every loop, every customer node would be judged whether they can be served by drone. We calculate the saved cost if it can be accessed by the drone (Line 3). If one or more customers can be found, choose the most cost-saving customer and adjust two-echo routes to change the delivery way of the chosen customer (Line 
5). If not, end the loop (Line 7) and output the solution, which is a two-echelon route for the truck and drone (Line 10).

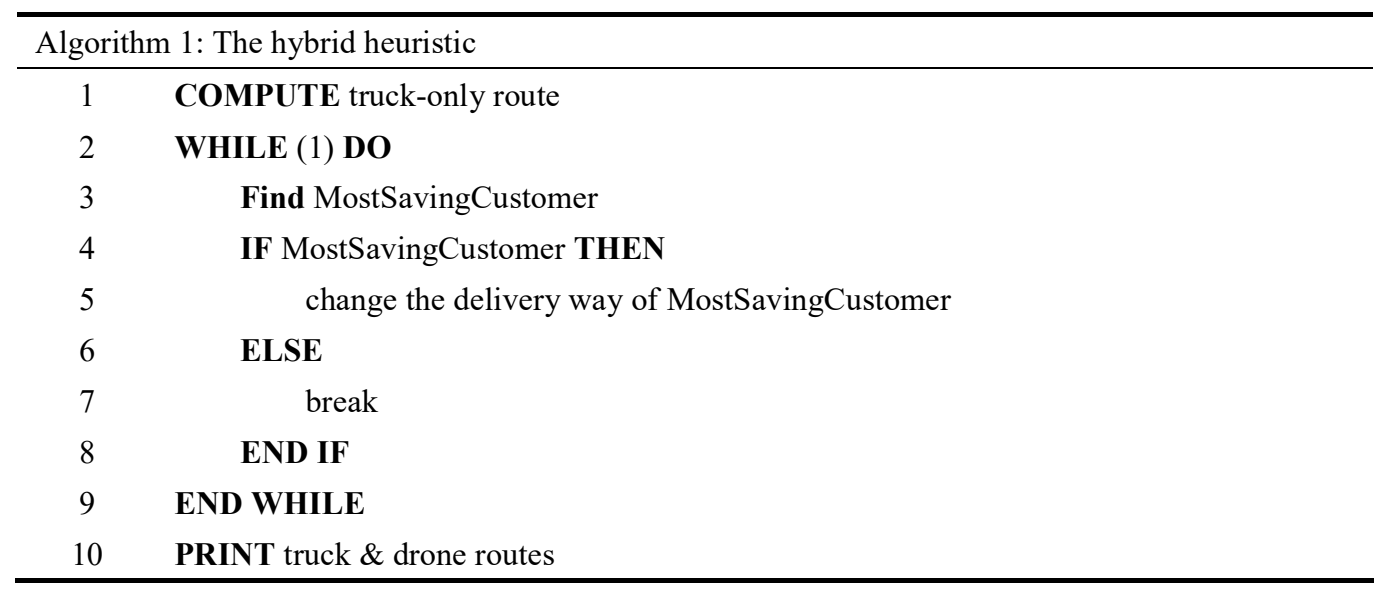

\subsection{Simulated Annealing Algorithm}

Simulated Annealing algorithm is proposed by Kirkpatrick et al. (1983). Since it accepts the worse solution with a certain probability, random factors are introduced into the search process and the global optimal solution would be possibly obtained. This algorithm provides an effective way for solving the TSP and VRP problems which are difficult to deal with by traditional methods (Reinelt, 1994). Simulated annealing is a heuristic based on an analogy of thermodynamics with the way metals cool and anneal. Its essential idea is not to restrict the search moves only to those solutions that decrease the objective function value, which can avoid being trapped prematurely in a local minimum. The main algorithm is shown below (Algorithm 2).

Initially, the optimization procedure starts from defining several parameters, such as the start temperature and the termination temperature (Line 1). After constructing the initial solution with the hybrid heuristic in 4.1 (Line 2), the initial solution would be assigned to the best solution for initialization and recorded as the current solution (Line 3). At each temperature, the number of iterations is predetermined and a Tabu list is initialized to improve the performance of simulated annealing in the inner loop (Line 5). Then in each iteration, the algorithm finds a temp solution from the neighborhood of the current solution (Line 8), which will be explained later in further detail. Also some invalid moves would be added into Tabu list (Line 8). If an improvement has been obtained (Line 10), the temp solution would become the 
current solution for the next iteration (Line 11). And it would remarked as the best solution if its objective is better than the best objective so far (Line 13). However, a worse solution may also be accepted with a small probability, determined by the current temperature and the objective fitness difference which is known as the Boltzmann function (Line 17). Under this condition, the best solution would not be changed. When the number of iterations reaches the termination conditions, the temperature would be cooled down (Line 22) and the Tabu list would be released (Line 23). As the current temperature is cooled to the end temperature, the algorithm is terminated and the best solution is outputted (Line 25).

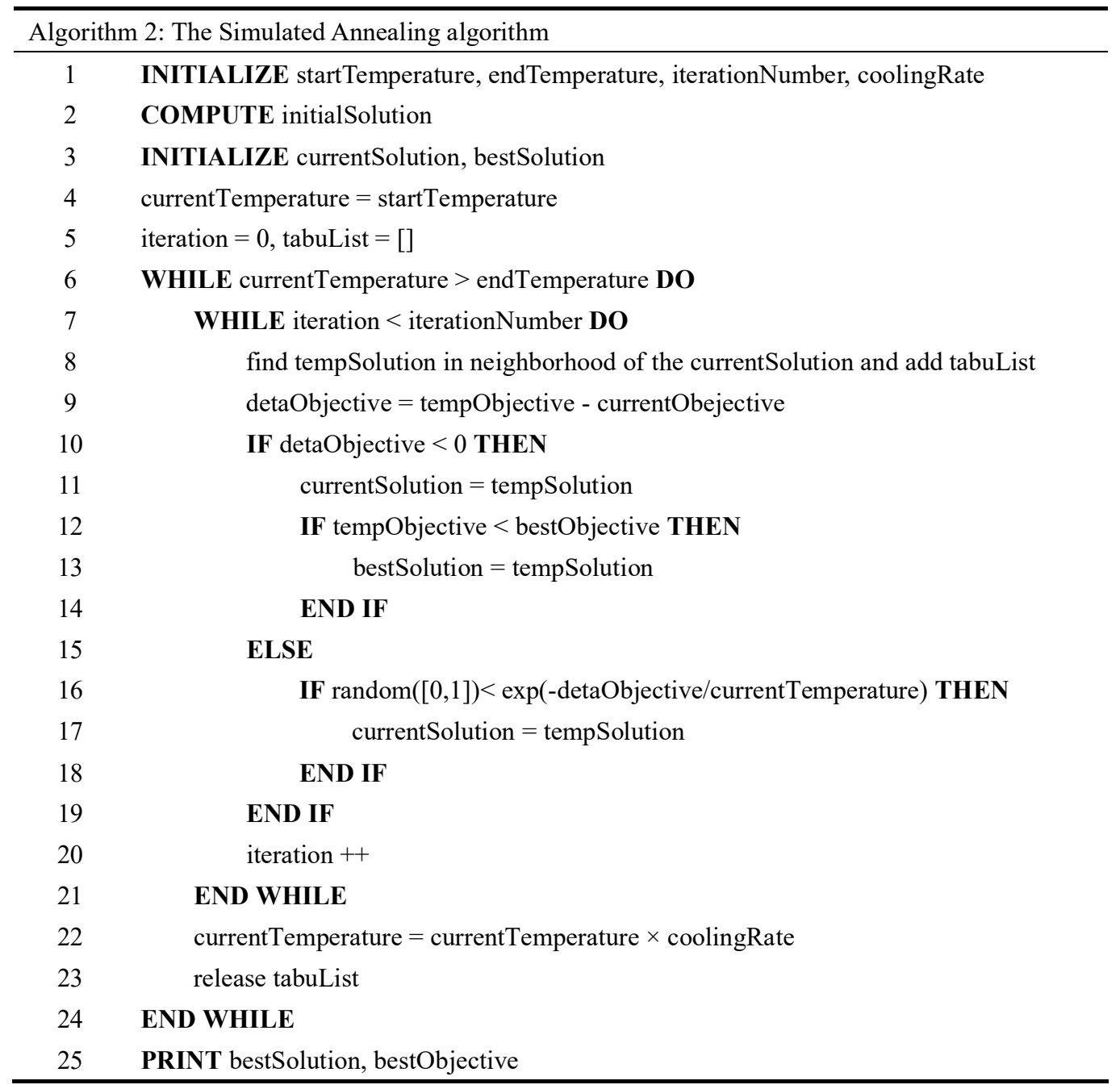

\subsubsection{Neighborhoods}

Since the two-echelon routes presents great complexity, it would produce many infeasible solutions with the traditional neighborhood structures used in TSP and VRP problems, which 
consumes large amount of useless computation. Thus, in this section, different situations are considered when the neighborhood move is performed in mixed customers and some adjustment is applied to ensure the feasibility of the solution. To be specific, three neighborhoods are designed. In each iteration, these neighborhoods of the current solution are all searched. And the generated feasible solutions would be compared to select the solution with the least cost.

\section{(1) Neighborhood 1: Deletion-Reinsertion}

The deletion-reinsertion neighborhood is commonly used in solving TSP related problems, which removes a customer and reinserts it in other position in a tentative solution. Different from traditional TSP problems, there are two kinds of routes, which are ground vehicle route and drone routes. We restrict that the deleted node can only be reinserted into the same type route, that is, the delivery tool of the parcel for the customers should not be changed. The removal move can either randomly select a customer or delete the customer that has the greatest impact on the distance of the route. Specifically, this move starts from generating a random number to determine the removal way. If it is removed based on the distance, the distance of both two sides of every customer would be calculated and the customer with the longest distance of two sides would be removed. However, if it is removed randomly, the search space for the solution would be diversified. As for the reinserting operation, it would greedily choose the best insertion position with a minimum increase in cost after considering all the feasibility.

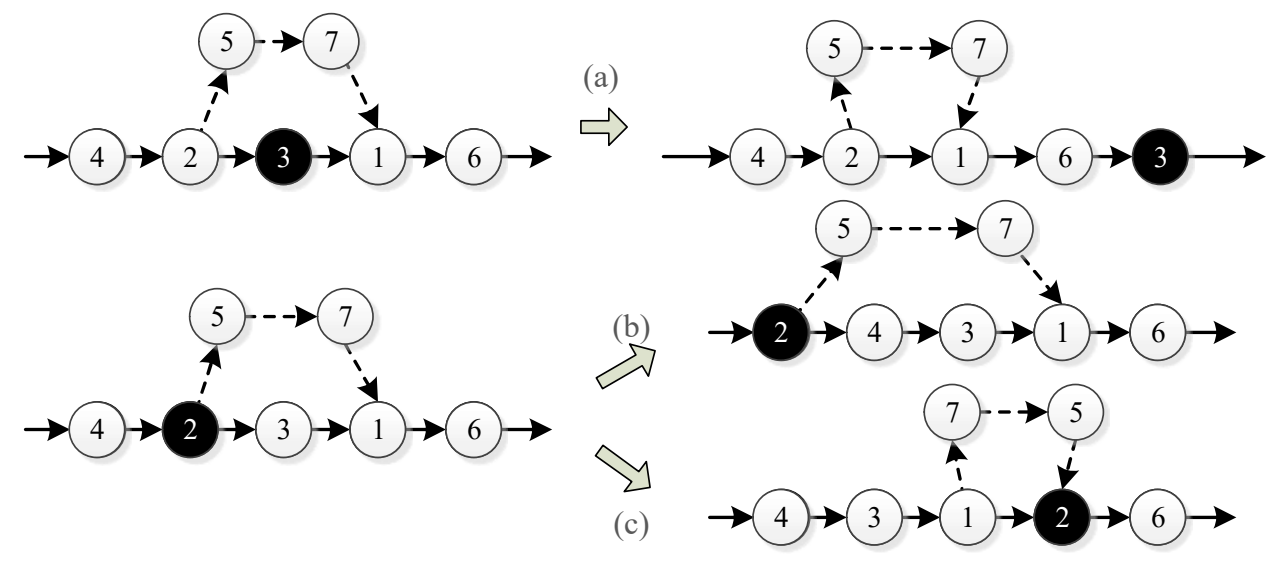

Figure 6. Sketch map for reinsertions of nodes visited by the truck

Since drone customers are only located on one drone route, the reinsertion of drone 
customers would only be limited to the endurance and capacity of the drone. Nevertheless, it is more complex for truck customers. Several typical operations for the deletion-reinsertion neighborhood is presented in Figure 6. Figure 6(a) illustrates a relatively straightforward move in which customer 3 is only located on the truck route and the reinsertion of customer 3 has no influence on the drone route. Additionally, the operations represented in Figure 6(b) and (c) relocate the customer 2, locating on both the truck route and a drone route. In case (b), although the truck route is relocated, the drone still launches from customer 2. However, in case (c) the drone route is inverted, turning customer 2 the return node of the drone route. According to the energy consumption model, the energy that the drone consumes on this route would change.

\section{(2) Neighborhood 2: 2-Exchange}

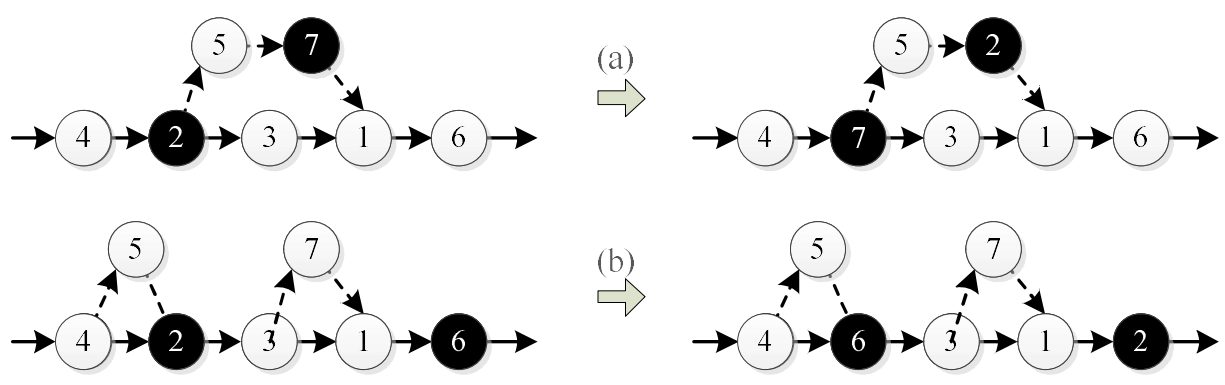

Figure 7. Sketch map for 2-exchange

The 2-exchange neighborhood is to swap a customer with another one in a solution. Due to the complexity of the two-echelon routes, here the 2-exchange operation is more complex than that utilized in traditional TSP, and also the capacities on the drone's endurance and payload should be checked in each operation. The exchange between two nodes in a single type route, e.g. the truck route or the drone route, is simple if the structure the two-echelon routes are not changed. In other cases, if the truck visiting node serves as the launch or landing node, the role of this node in the drone route should be replaced by the other exchanging node. Some special cases are presented in Figure 7. As shown in Figure 7(a), the departure node of the drone changes from customer 2 to customer 7, and the new route of the drone needs to be verified. In Figure (b), if the launch and return customers remain the same, the drone route $(3,7,1)$ would be inside another drone route $(4,5,2)$. Thus, it is supposed to do some adjustment after exchange and choose customer 6 to be the new returning customer. 


\section{(3) Neighborhood 3: Relocation}

The relocation neighborhood is similar with the deletion-reinsertion neighborhood, and their main difference is that the relocation must change the delivery way of customers. It is simple for drone visiting customers to be changed into truck visiting ones, so we will focus on how to turn truck visiting customers into drone visiting customers. Figure 8 presents three typical moves for relocation nodes between truck route and drone route. After removing a customer from the route of the truck, the simplest way is inserting it in a new drone trip as illustrated in Figure 8(a). Also, as Figure 8(b) presents, inserting the customer into current drone route is another method. Comparing between two possible solutions, the better one with lower cost would be chosen.
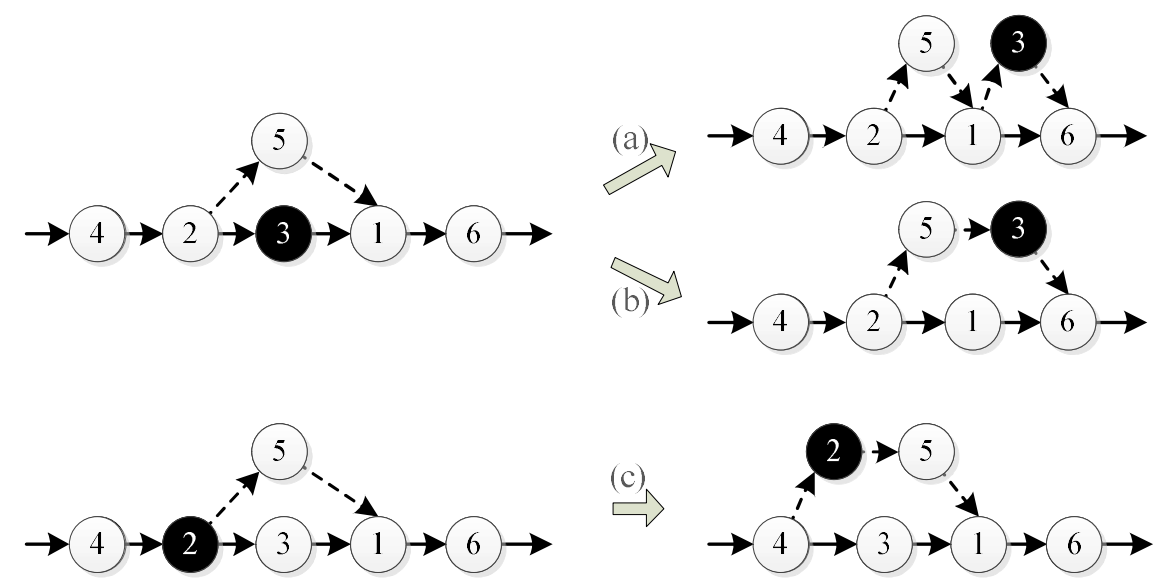

Figure 8. Sketch map for some cases of relocation between truck and drone nodes

It should be noted that if the selected truck customer is the launch node or landing node, an alternative customer is required after converting the selected customer to the drone customer. For instance, to ensure the feasibility of the solution, another customer should be chosen to be the launch of the drone after customer 2 is visited by drone, such as customer 4 in Figure 8(c).

\subsubsection{Integration of Tabu list}

Integrated with the transition probability, the simulated annealing algorithm has the capability to escape from local optima in the solution search process. However, the lack of memory may be regarded as the main deficiency of this method, which would result in shortterm cycling and revisiting (El-Bouri et al., 2007). Thus, with memorizing a list of forbidden moves (Tabu list), the performance of simulated annealing can be improved. And the integration 
of Tabu list into the simulated annealing algorithm has been reported to be effective in previous study (Wu et al., 2014).

In each interaction, some neighborhoods would be selected to generate a new neighborhood solution. If the new solution is better (with lower cost) than the old one, then it would be accepted. Or if the new solution has worse objective fitness than the old one, the new one can be accepted with a certain probability. Once the new solution has been accepted, selected neighborhoods would be added to the Tabu list. Consistently, neighborhoods in the Tabu list are not allowed to be inserted into the new solution until released after the temperature cools down.

\section{Experiments and Results}

In this section, experiments based on randomly generated instances are carried out to test the effectiveness of the proposed algorithms and a practical case is applied for the sensitivity analysis on some critical factors, which are the ratio of light parcels, drone's capacity on payload, and the capacity of drone's battery power.

\subsection{Experiment on random instances}

\subsubsection{Experiment Design}

The value of parameters related with the truck and drone is set according to typical ones in practical use. The drone's weigh is about $2 \mathrm{~kg}$ with a maximum payload of $3 \mathrm{~kg}$ and its endurance distance is about 6 miles (about 10 kilometers) under maximum payload condition. As for the truck cost, a typical truck for parcel delivery travels 6 miles per gallon of diesel, which yields that the fuel consumption is $0.392 \mathrm{~L} / \mathrm{km}$. On January 8, 2018, the national average price of diesel is $\$ 2.996$ per gallon, which is $\$ 0.310 / \mathrm{km}$. Besides, it is estimated that the variable operating cost of trucks is $\$ 0.484 \mathrm{~km}-1$ after considering maintenance cost, depreciation cost and salaries (Cachon, 2014). Thus, the unit distance cost for the truck is $\$ 0.794 / \mathrm{km}$. When it comes to the drone cost, it is set by default that the truck's unit distance cost is 25 times the drone's unit distance cost (Ha et al., 2018). With the battery power 5000mAh and the maximum travel distance 10 kilometers, it can be estimated that the unit energy cost for the drone is $\$ 0.635$ * $10^{\wedge}(-4) / \mathrm{mAh}$. Thus, after referring to public parameters of various drones, the performance of two vehicles is initially determined and reported in Table 3. 
Table 2. The Parameters of Truck and Drone

\begin{tabular}{lcc}
\hline \multirow{2}{*}{ Truck } & speed & $50 \mathrm{~km} / \mathrm{h}$ \\
& unit distance cost & $\$ 0.794 / \mathrm{km}$ \\
\hline \multirow{2}{*}{ Drone } & payload capacity & $3 \mathrm{~kg}$ \\
& maximum rate of power & $160 \mathrm{~W}$ \\
& battery power & $5000 \mathrm{mAh}$ \\
& coefficient $k=370 \eta \gamma(P-e)$ & 1280 \\
\hline
\end{tabular}

The random experiment is conducted with instances in three different scales which is displayed in Table 4. When generating the position of nodes, the quartering method is used as dividing the map into four parts and randomly generating the equal number of nodes in each part. As for the weight of parcels, Amazon has announced that about $90 \%-95 \%$ of its parcels are no heavier than 5 pounds (about $2.27 \mathrm{~kg}$ ). Thus, with some prior research, the weight of parcel is preset in the range of $0-10 \mathrm{~kg}$ and the parcels with the weight lighter than $2.3 \mathrm{~kg}$ accounts for 90 percent. If the parcel is too heavy to be delivered by drone, it is thought as a heavy parcel, otherwise a light parcel.

Table 3. Instances in Three Different Scales

\begin{tabular}{ccc}
\hline Scale & Number of Nodes & Map size \\
\hline Small & 20 & $8 \mathrm{~km} * 8 \mathrm{~km}$ \\
Medium & 40 & $10 \mathrm{~km}^{*} 10 \mathrm{~km}$ \\
Large & 100 & $12 \mathrm{~km}^{*} 12 \mathrm{~km}$ \\
\hline
\end{tabular}

Figure 9 presents an example of small scale instance. The depot is represented by triangle block. The dots indicate all the customers with the size distinguishing the heavy parcel and light parcel.

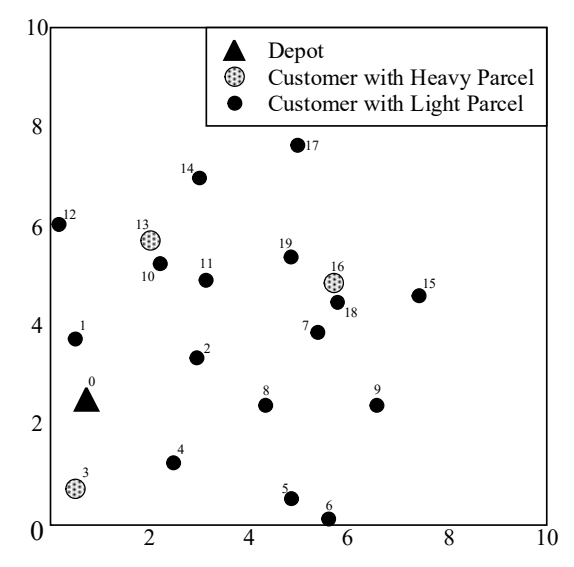

Figure 9. An example distribution of nodes for small scale instance 


\subsubsection{Experiment Result}

To explore the performance of the algorithm, ten instances for each different scale are randomly generated by the method described above and used to conduct the experiment. For every instance, the results for the initial solution obtained by the hybrid heuristic ( $\mathrm{HH})$ and the final impoved solution obtianed by the simulated alnnealing algorithm (SA) are compared.

In addition, the result for the truck-only route of each instance (TO) is calculated by the simulated annealing algorithm, which can be seen as the optimal solution if the parcels are delivered only by traditional truck. Then the comparison between final solution (SA) and the optimal truck-only solution (TO) is also presented, which demonstrates the impact on the total cost with the drone assisted mode.

Table 5 presents the computational results for all ten instances of small scale. We can see that the SA algorithm can significantly improve the initial solutions obtained by the $\mathrm{HH}$ algorithm, and the costs of improved solution presented by SA decrease from $13.47 \%$ to $57.14 \%$ comparing to the costs of the initial solutions by $\mathrm{HH}$. With the drone introduced, the final costs obtained by SA for the truck-drone delivery mode decrease from $20.92 \%$ to $65.63 \%$ comparing to the costs of truck-only mode. The computational time for all small instances is controlled in 12 seconds.

Table 4. Results of 10 Random Instances in Small Scale

\begin{tabular}{|c|c|c|c|c|c|c|}
\hline \multirow[b]{2}{*}{$\begin{array}{l}\text { Map } \\
\text { Size }\end{array}$} & \multicolumn{3}{|c|}{ Cost $/ \$$} & \multicolumn{2}{|c|}{ Comparison } & \multirow[b]{2}{*}{$\begin{array}{c}\text { Computational } \\
\text { Time (s) }\end{array}$} \\
\hline & $\begin{array}{l}\text { Truck- } \\
\text { Only } \\
\text { (TO) }\end{array}$ & $\begin{array}{c}\text { Truck \& } \\
\text { Drone } \\
(\mathrm{HH})\end{array}$ & $\begin{array}{c}\text { Truck \& } \\
\text { Drone } \\
\text { (SA) }\end{array}$ & $(\mathrm{HH}-\mathrm{SA}) / \mathrm{HH}$ & $(\mathrm{TO}-\mathrm{SA}) / \mathrm{TO}$ & \\
\hline \multirow{10}{*}{$\begin{array}{l}\text { Small } \\
\text { Scale }\end{array}$} & 58.36 & 45.74 & 27.47 & $39.94 \%$ & $52.93 \%$ & 10.340 \\
\hline & 47.14 & 37.66 & 20.33 & $46.02 \%$ & $56.87 \%$ & 9.015 \\
\hline & 61.08 & 51.24 & 27.82 & $45.71 \%$ & $54.45 \%$ & 8.868 \\
\hline & 56.39 & 45.32 & 27.59 & $39.12 \%$ & $51.07 \%$ & 10.961 \\
\hline & 52.20 & 45.25 & 32.86 & $27.38 \%$ & $37.05 \%$ & 9.504 \\
\hline & 58.61 & 35.93 & 26.50 & $26.25 \%$ & $54.79 \%$ & 11.952 \\
\hline & 55.01 & 50.27 & 43.50 & $13.47 \%$ & $20.92 \%$ & 7.693 \\
\hline & 52.66 & 48.86 & 32.95 & $32.56 \%$ & $37.43 \%$ & 7.810 \\
\hline & 57.36 & 53.06 & 26.64 & $49.79 \%$ & $53.56 \%$ & 8.353 \\
\hline & 48.82 & 39.15 & 16.78 & $57.14 \%$ & $65.63 \%$ & 9.278 \\
\hline
\end{tabular}

Table 6 presents the results for ten instances of medium scale. We can see that the solutions obtained by HH can be improved by SA for $27.35 \%$ - 49.33\%. The final costs for the truckdrone delivery mode decrease for $38.03 \%$ - 54.72\% comparing to the costs of truck-only mode. 
The computational time of SA for the medium instances is a little more than that for the small instances, which is still controlled in 23 seconds.

\begin{tabular}{|c|c|c|c|c|c|c|}
\hline \multirow[b]{2}{*}{$\begin{array}{l}\text { Map } \\
\text { Size }\end{array}$} & \multicolumn{3}{|c|}{ Cost $/ \$$} & \multicolumn{2}{|c|}{ Comparison } & \multirow[b]{2}{*}{$\begin{array}{l}\text { Computational } \\
\text { Time (s) }\end{array}$} \\
\hline & $\begin{array}{l}\text { Truck- } \\
\text { Only } \\
\text { (TO) }\end{array}$ & $\begin{array}{c}\text { Truck \& } \\
\text { Drone } \\
(\mathrm{HH}) \\
\end{array}$ & $\begin{array}{c}\text { Truck \& } \\
\text { Drone } \\
(\mathrm{SA})\end{array}$ & $(\mathrm{HH}-\mathrm{SA}) / \mathrm{HH}$ & (TO-SA)/TO & \\
\hline & 84.39 & 75.40 & 38.21 & $49.32 \%$ & $54.72 \%$ & 20.426 \\
\hline & 85.86 & 71.36 & 39.95 & $44.02 \%$ & $53.47 \%$ & 18.594 \\
\hline & 98.13 & 83.26 & 57.04 & $31.49 \%$ & $41.87 \%$ & 18.909 \\
\hline & 88.14 & 75.18 & 54.62 & $27.35 \%$ & $38.03 \%$ & 16.859 \\
\hline Med & 98.28 & 85.03 & 52.73 & $37.99 \%$ & $46.35 \%$ & 19.309 \\
\hline \multirow[t]{5}{*}{ Scale } & 98.67 & 75.87 & 53.89 & $28.97 \%$ & $45.38 \%$ & 22.829 \\
\hline & 95.45 & 77.55 & 44.02 & $43.24 \%$ & $53.88 \%$ & 18.463 \\
\hline & 102.21 & 86.19 & 52.82 & $38.72 \%$ & $48.32 \%$ & 20.679 \\
\hline & 94.95 & 71.94 & 51.16 & $28.89 \%$ & $46.12 \%$ & 17.417 \\
\hline & 83.82 & 69.30 & 49.64 & $28.37 \%$ & $40.78 \%$ & 19.082 \\
\hline
\end{tabular}

Table 7 shows the results for ten instances of large scale. It can be seen that the computational time for the large instances is much longer than that for the medium instances, while it is acceptable and controlled in 62 seconds. The performance of SA on solution improvement for large instances is similar to that of medium instances.

Table 6. Results of 10 Random Instances in Large Scale

\begin{tabular}{|c|c|c|c|c|c|c|}
\hline \multirow[b]{2}{*}{$\begin{array}{l}\text { Map } \\
\text { Size }\end{array}$} & \multicolumn{3}{|c|}{ Cost $/ \$$} & \multicolumn{2}{|c|}{ Comparison } & \multirow[b]{2}{*}{$\begin{array}{c}\text { Computational } \\
\text { Time (s) }\end{array}$} \\
\hline & $\begin{array}{l}\text { Truck- } \\
\text { Only } \\
\text { (TO) }\end{array}$ & $\begin{array}{c}\text { Truck \& } \\
\text { Drone } \\
(\mathrm{HH})\end{array}$ & $\begin{array}{c}\text { Truck \& } \\
\text { Drone } \\
\text { (SA) }\end{array}$ & $(\mathrm{HH}-\mathrm{SA}) / \mathrm{HH}$ & (TO-SA)/TO & \\
\hline & 184.05 & 150.08 & 99.64 & $33.61 \%$ & $45.86 \%$ & 61.279 \\
\hline & 173.21 & 146.02 & 102.79 & $29.61 \%$ & $40.66 \%$ & 57.073 \\
\hline & 170.53 & 136.21 & 94.27 & $30.79 \%$ & $44.72 \%$ & 53.689 \\
\hline & 188.55 & 170.21 & 101.80 & $40.19 \%$ & $46.01 \%$ & 47.574 \\
\hline Large & 170.94 & 150.85 & 93.44 & $38.06 \%$ & $45.34 \%$ & 53.540 \\
\hline \multirow[t]{5}{*}{ Scale } & 181.98 & 172.02 & 82.87 & $51.83 \%$ & $54.46 \%$ & 42.790 \\
\hline & 191.25 & 159.25 & 85.27 & $46.46 \%$ & $55.41 \%$ & 56.360 \\
\hline & 186.92 & 160.30 & 91.31 & $43.04 \%$ & $51.15 \%$ & 49.839 \\
\hline & 163.57 & 146.10 & 84.87 & $41.91 \%$ & $48.11 \%$ & 50.131 \\
\hline & 201.65 & 179.79 & 82.41 & $54.16 \%$ & $59.13 \%$ & 57.998 \\
\hline
\end{tabular}

From the overall results presented in Table 4-6, it can be observed that the adoption of cooperated truck and drone for parcel delivery can greatly reduce the delivery costs, compared with the truck-only mode. In many instances, the costs of the truck-drone mode are even less 
than the half of that with truck only. Besides, the neighborhood operators have also proven to be an effective local optimization method, and for most of the instances, the costs of the initial solution obtained by $\mathrm{HH}$ is reduced by SA for over $30 \%$.

Furthermore, the performance of the whole algorithm is more stable with larger scale instances. In small-scale instances, the distribution of customers is relatively scattered. At this time, the optimization process is directly affected by the layout of the nodes, which is the reason why the optimization proportions in Table 4 differ greatly. The density of customers would increase as enlarging the scale, which is more favorable to the drone delivery.

\subsection{Case Study}

\subsubsection{Case description}

A case is built based on the practical road network and customer nodes in Changsha, China, which is used for the sensitivity analysis. As Figure 10 displays, in the east urban area of Changsha, 95 crossings of the main roads are drawn and 30 customer nodes are marked. Through connecting these crossings, there forms a road network for the delivery truck.

Specifically, the latitude and longitude coordinates of the crossings are obtained from the Baidu map. As for the road distance of the truck, a tool provided by Baidu is applied for the distance calculation of the actual road segments, generating the initial distance matrix. Then the crossing distance matrix is filled by the Floyd algorithm to acqure the shortest distance between any two crossings based on the road network. The weight for each customer is randomly generated with the mentioned weight ratio, $90 \%$.

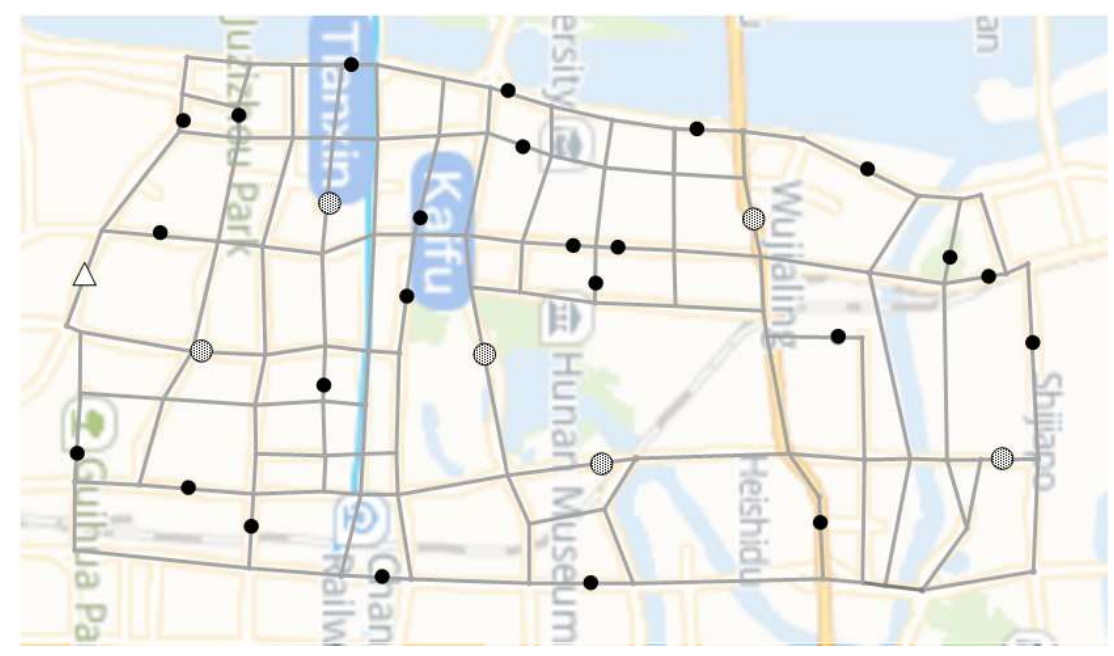

Figure 10. The layout of road network and the instance for 30 customers in Changsha 
To display the result visually, the basic simulated annealing algorithm and the constructed algorithm are both applied to solve the example instance in Figure 10 and the result is shown in Figure 11. Figure 11(a) presents the optimal truck-only solution with the cost of 28.63 while (b) is the final truck \& drone solution with the cost of 16.72. In the final solution, there are 10 customers delivered by the truck, including 6 customers with heavy parcels. The other 20 customers are visited by drone, which vastly reduces the delivery cost. And the computional time is 9.39 seconds.

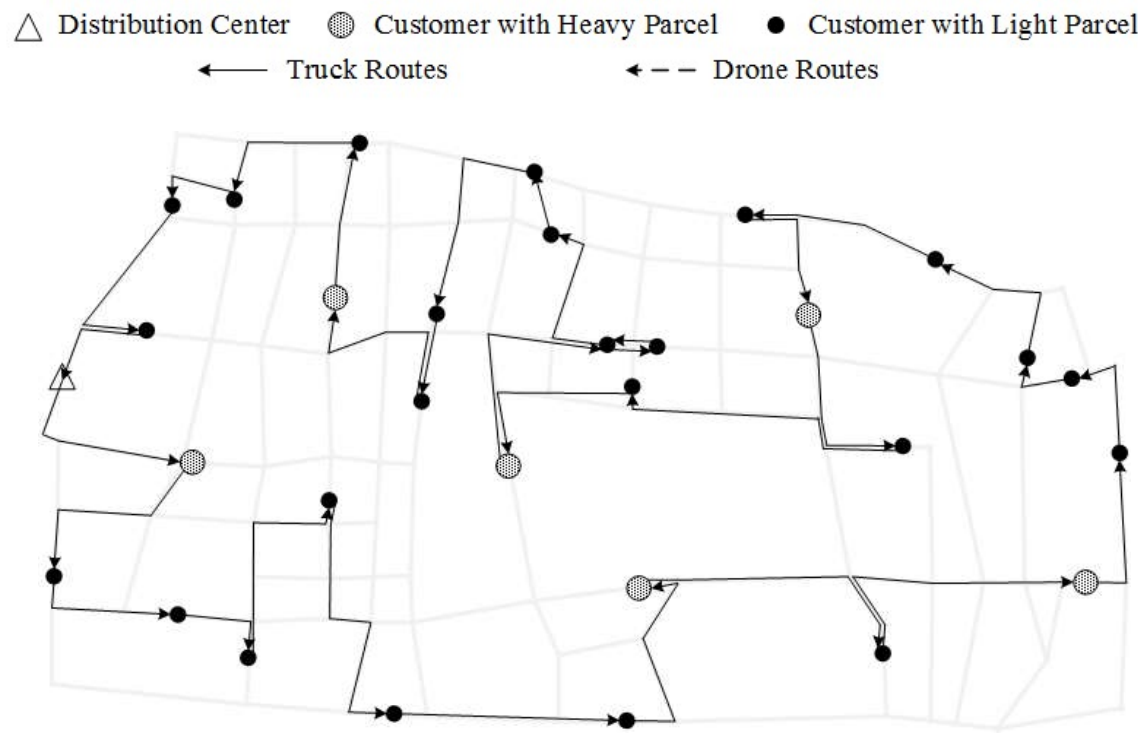

(a) the truck-only solution

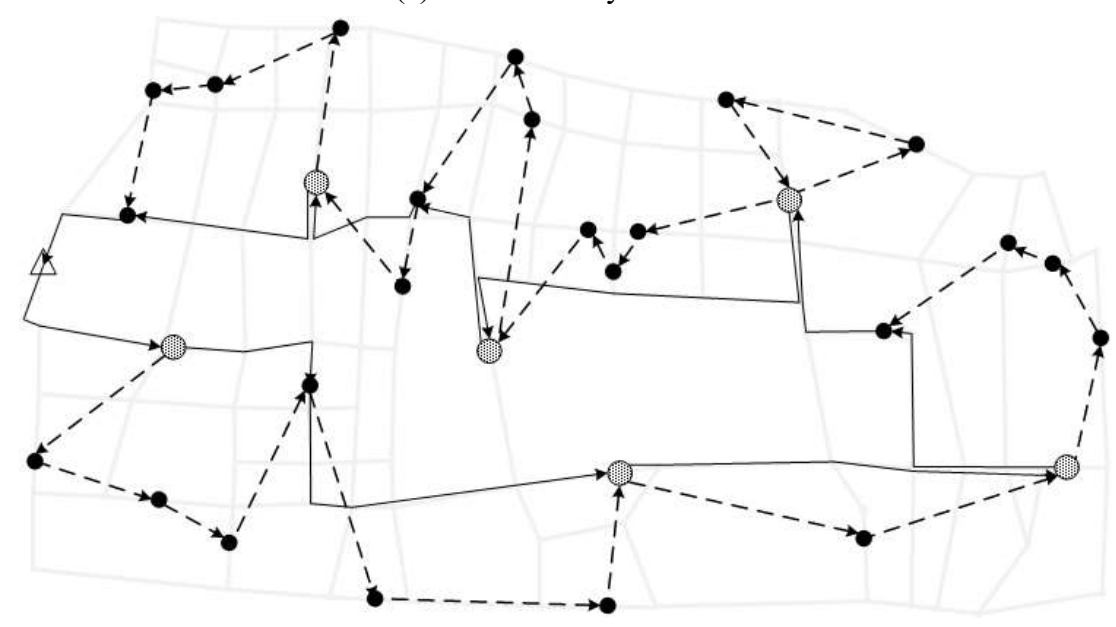

(b) final truck \& drone solution

Figure 11. Comparison of solutions for different delivery modes

\subsubsection{Sensitivity analysis}

Three critical factors are considered with sensitivity tests, which are the ratio of light parcels, drone's capacity on payload, and the capacity of drone's battery power. 


\section{(1) Impact analysis for the Ratio of light parcels}

When generating the weight of customers' parcels, a ratio of light parcels is defined, which means the percentage of light parcels under $2.3 \mathrm{~kg}$ (about 5 pounds) in all parcels. To monitor the effect of this ratio, the comparison experiments are conducted with different ratios from 0.50 to 0.95 . The results are presented in Figure 12 .

As the ratio of light parcel increases, the total cost shows an obvious reduction from 29.07 to 16.63 . And the cost generated by the truck also falls from 24.69 to 8.30 while the cost generated by the drone rises from 4.38 to 8.33 . It can be inferred that with the proportion of parcels within the maximum payload of drone rises, more customers would be served by the drone, which can save the overall delivery cost. Furthermore, when the ratio of package is larger than 0.8 , the costs do not change a lot. At this time, the capacity of the payload no longer restricts the delivery of drone, but the endurance capacity becomes the main constraint.

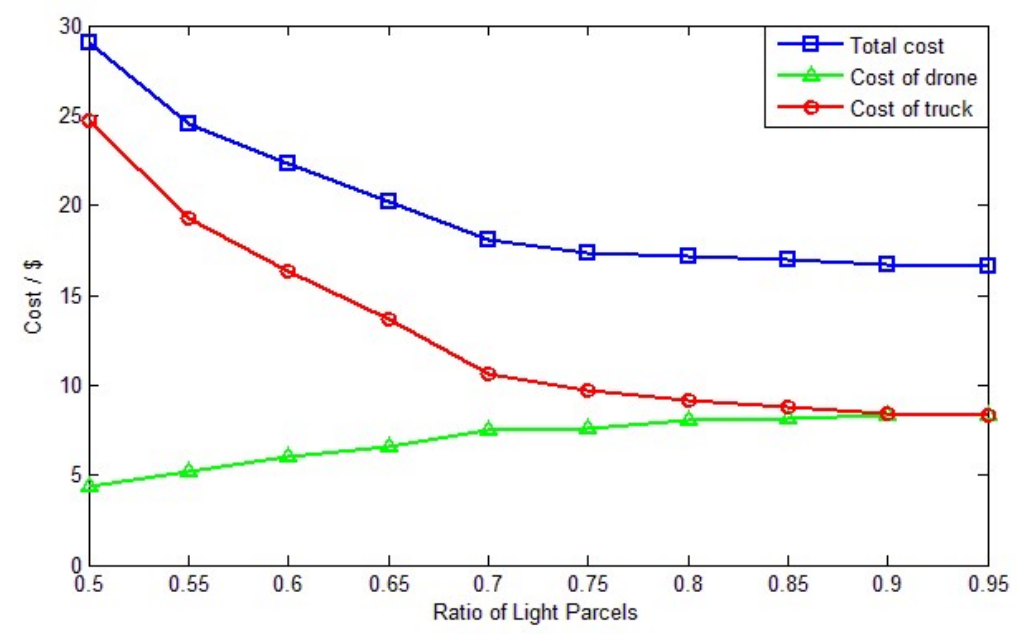

Figure 12. Computational results under different ratios of light parcels

\section{(2) Impact analysis of the drone's capacity on payload}

The method of increasing the drone's capacity on payload depends on the same ides with the way of improving the ratio of light parcels, which can promote the utilizing efficiency of the drone. Thus, the result of changing the payload from $2 \mathrm{~kg}$ to $8 \mathrm{~kg}$ is similar to the above test, which are shown in Figure 13.

It is obvious that both total cost and truck cost experienced a significant decline as the drone payload capacity rises, especially from $2.0 \mathrm{~kg}$ to $3.0 \mathrm{~kg}$. The total cost decreases from 23.16 to 14.27 and the reduction is even bigger for the truck cost from 18.78 to 5.33. It can be noticed 
that the drone cost would be greater than the truck cost with maximum payload above $3 \mathrm{~kg}$, which can be inferred that the drone delivery would play a major role as long as it gets rid of the limitations of the payload capacity. However, when the maximum drone payload exceeds $5.0 \mathrm{~kg}$, the continuous increase of the maximum payload will not lead to a significant reduction in the total cost. That is because the constraints on the drone endurance restricts it to be used for delivering more parcels in one route.

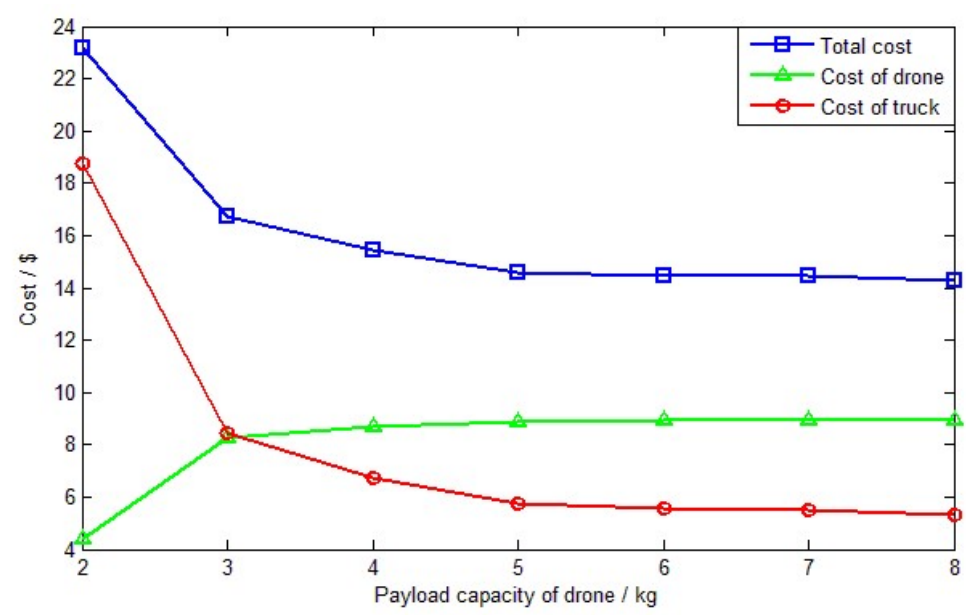

Figure 13. Computational results under different capacities of drone's payload

\section{(3) Impact analysis of the drone's battery power}

To investigate the effects of the capacity of drone's battery power, the sensitivity test is carried out in a way that the only difference is the battery power varying from 4000 to $8000 \mathrm{mAh}$.

As Figure 14 displays, when the capacity of the battery power increases from 3000 to $5500 \mathrm{mAh}$, the total cost reduces within a wide range from 29.16 to 15.41 . It is obvious that increasing battery power can be a valid way to reduce the delivery cost. As for the other part of the cost, the truck cost decreases a lot from 25.78 to 7.09 while the cost of drone increases from 3.38 to 8.32 . However, the total cost basically keeps the same when the capacity of battery is over $6000 \mathrm{mAh}$, since the endurance of drone would not be the primary limitation for drone delivery. In this situation, due to the limitation on drone's payload, the drone cannot serve more customers in one route, although the power is still sufficient. 


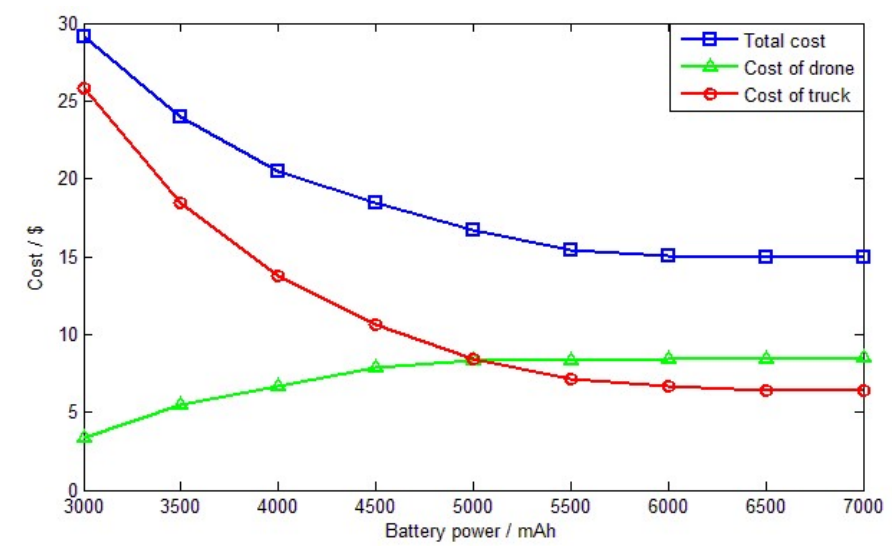

Figure 14. Computational results under different capacities of drone's battery power

\section{Conclusions}

In this paper, recognizing the importance and necessity of the coordination of the truck and the drone in parcel delivery, the drone-assisted parcel delivery problem is studied. Since multiple parcels are allowed to be delivered by the drone in one flight, the investigated problem can be viewed as a new variant of two-echelon routing problem. Besides, the effect of payload on the energy consumption is estimated by a proposed energy consumption model. Then an algorithm based on simulated annealing is presented. A heuristic combining Nearest Neighbor Algorithm and Saving Algorithm is designed to generate the initial solution. And variable local search is conducted with removal operators and insertion operator in every iteration to find better solution.

Radom test experiments with different scales are conducted and indicated the efficiency of the proposed algorithm. Furthermore, three factors are considered in sensitivity tests based on a practical case, which are the ratio of light parcels, maximum payload of drone, and drone's battery power. Experimental results show that the employment of the drone can save more costs when there are more light parcels for delivery. Also, proper improvement on technologies for enlarging the capacities of the drone's payload and battery power would help save more delivery cost.

It is an emerging phenomenon in recent years that the drone is employed in parcel delivery to cooperate with the truck. There are many research topics that are not studied in the management and operation research area, such as the two-echelon truck \& drone routing problems with time window, with multiple vehicles, and with multiple depots. More interesting and valuable variants of this problem are waiting to be explored. Due to the complexity for the optimization of the two-echelon routes, more efficient algorithms also have to be developed in future work. 


\section{Acknowledgments}

The research is supported by the National Natural Science Foundation of China (grant no. 71771215) and the Natural Science Fund for Distinguished Young Scholars of Hunan Province (2018JJ1035).

\section{References}

Agatz, N., Bouman, P., \& Schmidt, M. (2018). Optimization approaches for the traveling salesman problem with drone. Transportation Science.

Avellar, G. S., Pereira, G. A., Pimenta, L. C., \& Iscold, P. (2015). Multi-UAV routing for area coverage and remote sensing with minimum time. Sensors, 15(11), 27783-27803.

Cachon, G. P. (2014). Retail store density and the cost of greenhouse gas emissions. Management Science, 60(8), 1907-1925.

Choi, Y., \& Schonfeld, P. M. (2017, January). Optimization of multi-package drone deliveries considering battery capacity. In Proceedings of the 96th Annual Meeting of the Transportation Research Board, Washington, DC, USA (pp. 8-12).

Cover, T., \& Hart, P. (1967). Nearest neighbor pattern classification. IEEE transactions on information theory, 13(1), 21-27.

D'Andrea, R. (2014). Guest editorial can drones deliver?. IEEE Transactions on Automation Science and Engineering, 11(3), 647-648.

Dorling, K., Heinrichs, J., Messier, G. G., \& Magierowski, S. (2017). Vehicle routing problems for drone delivery. IEEE Transactions on Systems, Man, and Cybernetics: Systems, 47(1), 70-85.

El-Bouri, A., Azizi, N., \& Zolfaghari, S. (2007). A comparative study of a new heuristic based on adaptive memory programming and simulated annealing: The case of job shop scheduling. European Journal of Operational Research, 177(3), 1894-1910.

Freitas, J. C., \& Penna, P. H. V. (2018). A Randomized Variable Neighborhood Descent Heuristic to Solve the Flying Sidekick Traveling Salesman Problem. Electronic Notes in Discrete Mathematics, 66, 95102.

Grocholsky, B., Keller, J., Kumar, R. V., \& Pappas, G. J. (2006). Cooperative air and ground surveillance.

Ham, A. M. (2018). Integrated scheduling of m-truck, m-drone, and m-depot constrained by timewindow, drop-pickup, and $\mathrm{m}$-visit using constraint programming. Transportation Research Part C: Emerging Technologies, 91, 1-14.

Ha, Q. M., Deville, Y., Pham, Q. D., \& Ha, M. H. (2015). Heuristic methods for the traveling salesman problem with drone. http://ds.libol.fpt.edu.vn/handle/123456789/1994

Ha, Q. M., Deville, Y., Pham, Q. D., \& Hà, M. H. (2018). On the min-cost traveling salesman problem with drone. Transportation Research Part C: Emerging Technologies, 86, 597-621.

Kirkpatrick, S., Gelatt, C. D., \& Vecchi, M. P. (1983). Optimization by simulated annealing. science, 220(4598), 671-680.

Lin, S. W., Vincent, F. Y., \& Chou, S. Y. (2009). Solving the truck and trailer routing problem based on a simulated annealing heuristic. Computers \& Operations Research, 36(5), 1683-1692.

Luo, Z., Liu, Z., \& Shi, J. (2017). A two-echelon cooperated routing problem for a ground vehicle and its carried unmanned aerial vehicle. Sensors, 17(5), 1144.

Maini, P., \& Sujit, P. B. (2015, June). On cooperation between a fuel constrained UAV and a refueling 
UGV for large scale mapping applications. In Unmanned Aircraft Systems (ICUAS), 2015 International Conference on (pp. 1370-1377). IEEE.

Manyam, S. G., Rasmussen, S., Casbeer, D. W., Kalyanam, K., \& Manickam, S. (2017, June). MultiUAV routing for persistent intelligence surveillance \& reconnaissance missions. In Unmanned Aircraft Systems (ICUAS), 2017 International Conference on (pp. 573-580). IEEE.

Mufalli, F., Batta, R., \& Nagi, R. (2012). Simultaneous sensor selection and routing of unmanned aerial vehicles for complex mission plans. Computers \& Operations Research, 39(11), 2787-2799.

Murray, C. C., \& Chu, A. G. (2015). The flying sidekick traveling salesman problem: Optimization of drone-assisted parcel delivery. Transportation Research Part C: Emerging Technologies, 54, 86-109.

Reinelt, G. (1994). The traveling salesman: computational solutions for TSP applications. SpringerVerlag.

San, K. T., Lee, E. Y., \& Chang, Y. S. (2016, October). The delivery assignment solution for swarms of UAVs dealing with multi-dimensional chromosome representation of genetic algorithm. In Ubiquitous Computing, Electronics \& Mobile Communication Conference (UEMCON), IEEE Annual (pp. 1-7). IEEE.

Savuran, H., \& Karakaya, M. (2015). Route optimization method for unmanned air vehicle launched from a carrier. Lecture Notes on Software Engineering, 3(4), 279.

Shetty, V. K., Sudit, M., \& Nagi, R. (2008). Priority-based assignment and routing of a fleet of unmanned combat aerial vehicles. Computers \& Operations Research, 35(6), 1813-1828.

Song, B. D., Park, K., \& Kim, J. (2018). Persistent UAV delivery logistics: MILP formulation and efficient heuristic. Computers \& Industrial Engineering, 120, 418-428.

Sundar, K., \& Rathinam, S. (2014). Algorithms for Routing an Unmanned Aerial Vehicle in the Presence of Refueling Depots. IEEE Trans. Automation Science and Engineering, 11(1), 287-294.

Vincent, F. Y., Lin, S. W., Lee, W., \& Ting, C. J. (2010). A simulated annealing heuristic for the capacitated location routing problem. Computers \& Industrial Engineering, 58(2), 288-299.

Wu, G., Wang, H., Li, H., Pedrycz, W., Li H., Wang L. (2017). An adaptive Simulated Annealing-based satellite observation scheduling method combined with a dynamic task clustering strategy.Computers and Industrial Engineering, 113: 576-588. 